\title{
Preface
}

\section{NON-CLASSICAL MODELS OF AUTOMATA AND APPLICATIONS VII}

The Seventh Workshop on Non-Classical Models of Automata and Applications (NCMA 2015) was organised to bring together researchers who work on various aspects of non-classical and classical models of automata, providing an excellent opportunity to develop and discuss novel ideas. Numerous models of automata, both classical and non-classical, are natural objects of theoretical computer science. They are studied from different perspectives in various areas, both as theoretical concepts and as formal models for applications. The purpose of the NCMA workshop series is to promote a deeper and interdisciplinary coverage of this particular area and in this way foster new insights and substantial progress in computer science as a whole.

Previous NCMA workshops were held in Wrocław (2009), Jena (2010), Milano (2011), Fribourg (2012), Umeå (2013) and Kassel (2014). The Seventh Workshop on Non-Classical Models of Automata and Applications (NCMA 2015) took place in Porto, Portugal, on August 31st and September 1st, 2015. It was partially supported by the University of Porto and the Fundação para a Ciência e Tecnologia (FCT). We warmly thank the invited speakers Patricia Boyer-Decitre (LSV, CNRS and ENS de Cachan, France), Sylvain Lombardy (LaBRI, Bordeaux, France) and Emanuele Rodaro (CMUP, University of Porto, Portugal).

For NCMA 2015, we received submissions by a total of 22 authors from 8 different countries. From these submissions, on the basis of three referees' reports each, the Program Committee selected 9 contributions for presentation at NCMA. Also 4 short contributions were presented at the workshop. We thank the members of the Program Committee for their excellent work in making this selection: Maria Paola Bianchi (ETH Zürich, Switzerland), Henning Bordihn (Universität Potsdam, Germany), Cezar Câmpeanu (University of Prince Edward Island, PEI, Canada), Dominik D. Freydenberger (Universität Frankfurt, Germany), Rudolf Freund (Technical University, Vienna, Austria), Mika Hirvensalo (University of Turku, Finland), Markus Holzer (Universität Giessen, Germany), Jarkko Kari (University of Turku, Finland), António Machiavelo (University of Porto, Portugal), Carlo Mereghetti (University of Milano, Italy), Nelma Moreira (University of Porto, Portugal), František Mráz (Charles University at Prague, Czech Republic), Friedrich Otto (Universität Kassel, Germany), Rogério Reis (University of Porto, Portugal), Shinnosuke Seki (University of Tokyo, Japan), György Vaszil (University of Debrecen, Hungary), and Matthias Wendlandt (Universität Giessen, Germany).

We are grateful to the Organising Committee, the Centre of Mathematics of University of Porto and the Computer Science Department of Science Faculty of University of Porto for the support in the local organisation of NCMA 2015. Special thanks go to Rudolf Freund and the Institute of Computer Languages of the Vienna University for their assistance in editing the Proceedings which appeared as volume 318 in the series books@ocg.at of the Austrian Computer Society, and the booklet containing the short papers presented at NCMA 2015. 
The authors of some selected papers were invited to submit substantially enhanced versions of their papers to this special issue; each paper was reviewed and, if necessary, revised by the authors. We are grateful to all the authors for their contributions and all the referees for their reports and efforts. We would also like to express our thanks to Christian Choffrut and Olivier Serre, Editors-in-Chief of the journal, and to RAIRO-Theoretical Informatics and Applications Editorial Staff, in particular Muriel Bouquant, for their support to realize this special issue.

Special issue editors

Markus Holzer

holzer@informatik. uni-giessen.de

Nelma Moreira

nam@dcc.fc.up.pt

Rogério Reis

rvr@dcc.fc.up.pt 